\title{
Compensation technique for environmental and light source power variations applied in a polymer optical fiber curvature sensor for wearable devices
}

\author{
Arnaldo Gomes Leal-Junior ${ }^{1 *}$, Anselmo Frizera ${ }^{1}$, Maria José Pontes ${ }^{1}$ \\ ${ }^{1}$ Graduate Program of Electrical Engineering, Federal University of Espirito Santo, Vitória, ES, Brazil.
}

\begin{abstract}
Introduction: Polymer optical fibers (POF) are lightweight, present high elastic strain limits, fracture toughness, flexibility in bend, and are not influenced by electromagnetic fields. These characteristics enable the application of POF as curvature sensor and can overcome the limitations of the conventional technologies, especially for wearable and soft robotics devices. Nevertheless, POF based curvature sensors can suffer from environmental and light source power deviations. This paper presents a compensation technique for the environmental and light source power deviations in a POF curvature sensor. Methods: The curvature sensor was submitted to variations of temperature, humidity and light source power to characterize the sensor response and evaluate the proposed compensation technique. In addition, tests with the simultaneous variation of the angle and light source power variation were performed. Results: Results show that temperature and humidity effects do not lead to significative errors on the sensor measurement for wearable devices application, where a hardware-based compact and portable compensation technique of the light source deviation is applied. Moreover, the sensor with the compensation technique developed is compared with a potentiometer for dynamic measurements and the root-mean-square error of about $1^{\circ}$ is obtained, which is lower than sensors based on similar operation principle presented in the literature and some commercially available devices. Conclusions: The compensation technique proposed was able to compensate power deviations applied and resulted in a sensor with low errors with the additional advantages of compactness and low-cost, which enable its application as wearable sensors and on the instrumentation of wearable robots.
\end{abstract}

Keywords Optical fiber sensors, Wearable devices, Angle measurement.

\section{Introduction}

Measurement of gait kinematics is an important tool to aid on pathologies diagnosis, surgical interventions, and rehabilitation exercises (Dejnabadi et al., 2005). Joint angles and positions may also be applied as a feedback information for neural prostheses control or functional electrical stimulation (FES) (Tong and Granat, 1999). Furthermore, the increasing level of competitiveness and science application in high performance sports enable

(c) (i) This is an Open Access article distributed under the terms of the Creative Commons Attribution License, which permits unrestricted use, distribution, and reproduction in any medium, provided the original work is properly cited.

How to cite this article: Leal-Junior AG, Frizera A, Pontes MJ. Compensation technique for environmental and light source power variations applied in a polymer optical fiber curvature sensor for wearable devices. Res Biomed Eng. 2018; 34(1):37-44. DOI: $10.1590 / 2446-4740.04917$.

*Corresponding author: Graduate Program of Electrical Engineering, Federal University of Espírito Santo, Fernando Ferrari Avenue, 514, CEP29075-910, Vitória, ES, Brazil. E-mail: arnaldo.leal@aluno.ufes.br Received: 18 August 2017 / Accepted: 03 February 2018 the application of joint angle assessment techniques for training athletes (Rhea et al., 2016).

Although there are several techniques for gait kinematics analysis, the video-based technique provides the most reliable results. This technique, however, is expensive, time-consuming, and limited to laboratory environment (El-Gohary and McNames, 2012). For these reasons, wearable and portable sensors have been proposed. One of the most popular technologies are the inertial measurement units (IMUs) that comprise of tri-axial gyroscopes, accelerometers, and magnetometers. Sensor fusion algorithms provide the sensor interaction and complementary information (El-Gohary and McNames, 2012). IMUs are employed in many applications, such as timed-up and go tests (Coulthard et al., 2015), sit-stand and step-up transfer tests (Bolink et al., 2016), evaluation of kinematics and the capacity of post-stroke patients to grasp objects (Braidot et al., 2013). However, it presents high sensitivity with magnetic field interferences and need frequent calibration (El-Gohary and McNames, 2012). Other common devices are the potentiometers that usually need mechanical supports precisely assembled 
to the joint, which are a widely applied technology to measure angles in active orthosis and exoskeletons (Moreno et al., 2008; Villa-Parra et al., 2015). However, it can result in a less compact and more intrusive system (Wang et al., 2011). Furthermore, their use may also result in a noisy measurement (Moreno et al., 2008). These disadvantages may also inhibit the application of conventional technologies for angle measurement in wearable devices and in soft robotics applications, which is a growing field of research that employs flexible materials or structures as active or semi-active actuators of robotic devices (Holland et al., 2017). Considering the flexible structures used in soft robotics, potentiometers (broadly used in active orthoses) may not be a suitable solution.

To overcome these limitations, polymer optical fibers (POF) sensors may be an interesting solution for curvature sensing. POFs are compact, lightweight, allow multiplexing systems and are not influenced by magnetic fields (Bilro et al., 2012). Moreover, the polymer higher strain limits and fracture toughness enable the fiber bent in angles with the same order of magnitude of the human joints range of movement (Peters, 2011). Different operation principles for POF sensors have been proposed throughout the years such as light coupling based sensors (Antunes et al., 2013; Zawawi et al., 2013), specklegrams (Rodriguez-Cobo et al., 2013), Fiber Bragg Gratings (Marques et al., 2015) and intensity variation detection (Bilro et al., 2011). Among them, intensity variation is one of the most commonly used principles for joint angle measurement due to its ease of implementation, good price-quality ratio, and simplicity in signal processing (Bilro et al., 2012).

The intensity modulation principle for angle assessment is the variation of the optical power with the fiber curvature due to radiation losses (Bilro et al., 2011). The power attenuation can be measured with photodiodes and is related with the fiber curvature angle. This operation principle is further discussed and modelled in Leal-Junior et al. (2017a), Liu et al. (2006), and is employed in Dunne et al. (2008) for monitoring the seated spinal posture. Furthermore, Williams et al. (2010) applies a POF curvature sensor for lumbar spine angle assessment and the results are compared with the video-based technique. To increase the sensor sensitivity with the curvature, Donno et al. (2008) employ paddles around which the fiber is looped. However, one of the most common technique to enhance sensor sensitivity is a lateral section on the curvature region of the POF sensor by removing part of the fiber core, which creates a sensitive zone where there is more signal attenuation due to macrobending (Liu et al., 2006).

Different authors have reported the influence of the environmental conditions on the POF response Bilro et al.
(2011), Peters (2011), Vallan et al. (2012), Zawawi et al. (2013). For this reason, the POF curvature sensor design commonly takes into account possible deviations due to temperature, humidity, and light source power deviations. Since these deviations can provide errors in measurement, some compensation techniques have been proposed. Yuan (1998) developed a compensation technique using a five-fiber structure on the sensing probe. Although this system can sense two-dimensional physical parameters and automatically compensate the light source deviations, the sensor does not present a compact design, which is desired for wearable sensors purposes. Vallan et al. (2012) developed a dual-wavelength compensation technique. This technique employs a red and a blue light source, which are coupled in a single fiber. There are a dichroic filter and a mirror at a fixed distance of the fiber end. The reflected red signal is used for displacement measurement, whereas the blue signal is applied for disturbances compensation. Although this solution provides reliable results, this technique needs two light sources, filters, and mirrors. These additional components make the sensor less portable, compact and can be seen as a complex setup for a portable and wearable solution. Zawawi et al. (2013) compensated the light power deviations from the light source with an aluminum foil placed in a defined distance of the source fiber tip. The reference signal was collected at the end of the reference fiber due to reflection of the aluminum foil surface. The signal is compared with the one of the source fiber and a compensated response of the output voltage is achieved. Nevertheless, the reference fiber signal has low intensity due to reflection losses.

Bilro et al. (2011) and Moraleda et al. (2013) employed a self-referencing system, which lowers the source power deviations to acceptable values. This system comprises of a sensor fiber and a reference fiber with the same properties as the sensor fiber in a static position. The sensor response is normalized with respect to the reference fiber. However, for a curvature sensor, this technique may not be feasible for portable and wearable devices, since it is difficult to place a static fiber to compensate the environmental and light source deviations on the curvature region. Therefore, if this technique is employed for angle assessment in wearable applications, there will be a curvature of both the sensor and the reference fiber, which leads to an attenuation of the reference fiber response that provoke errors on the angle measurement. For this reason, wearable curvature sensors based on POF generally not employ a compensation for the environmental and light source deviations as presented in (Donno et al., 2008; Stupar et al., 2012), which are sources of errors on these sensors applications. 
This paper aims to describe the development of a POF based curvature sensor with compensation of the environmental and light source power deviations for wearable devices, such as robotic exoskeletons, advanced orthotic devices, wearable sensors and soft robots. The developed sensor is based on a lateral section to enhance sensitivity and is designed to dynamically measure angles from $0^{\circ}$ up to $90^{\circ}$, which are within the range of gait analysis applications (Kirtley, 2006). To obtain a feasible compensation technique, it is necessary to evaluate the effects of the light source, temperature, and humidity deviations on the POF sensor response.

\section{Methods}

\section{Experimental setup}

The design of the sensor comprises of controlling the lateral section parameters, namely lateral section length, depth and surface roughness. The lateral section is made through abrasive removal of material. A sandpaper with controlled grit size is attached to a rotating tool, which is positioned on supports with controlled dimensions to create a sensitive zone with controlled length and depth. The controlled grit size of the sandpaper provides the desired surface roughness of the sample. The lateral section parameters of the sensor employed on these analyses are $15.05 \mathrm{~mm}$ of section length and its depth is $0.66 \mathrm{~mm}$, which provides high sensitivity for the sensor according to the analytical model presented in (Leal-Junior et al., 2017a). Furthermore, the lateral section was made through abrasive material removal by a sandpaper with 400 -grit size. The sensor performance is evaluated with dynamic tests on the test bench presented in Figure 1. The test prototype has a servomotor to bend the fiber dynamically on controlled positions and velocities. The laser and the light coupler are positioned on the fixed part. The photodiode with a transimpedance amplifier is placed on the movable part. Another board with a photodiode and a second

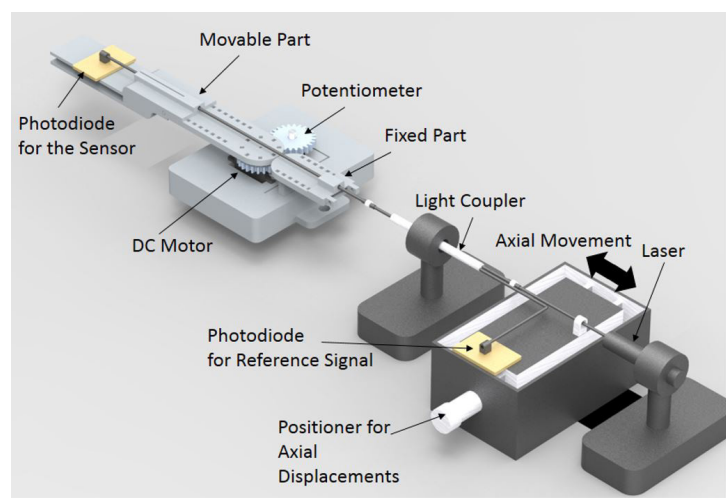

Figure 1. Test prototype for POF curvature sensor. transimpedance amplifier is placed on the fixed part of the test bench. By doing so, a compact design is achieved for the sensor compensation for power fluctuations. The axial displacement mechanism emulates the light source power deviations during the flexion/extension tests. A potentiometer is employed to measure the angular displacement of the movable part and its response is compared with the POF sensor response.

The velocity employed on the sensor tests is $37.20 \%$ and the test range is 0 to $90^{\circ}$ for flexion and extension. The angle range of the test as well as the angular velocity are within the range of human motion (Kirtley, 2006), where three consecutive cycles were made.

The light source is provided by a $3 \mathrm{~mW}$ red laser, the signal is divided to the reference fiber and the sensor through a 50:50 light coupler (LC). A photodiode (PD) IF-D91 (Industrial Fiber Optics, USA) with a transimpedance amplifier is employed to measure the sensor and the reference responses. The optical fiber employed is a multimode HFBR-EUS100Z POF (Broadcom Limited, Singapore) with step refractive index distribution. The POF employed has a polymethyl methacrylate (PMMA) core with diameter of $0.98 \mathrm{~mm}$ and the cladding is made of a fluorinated polymer with $1 \mathrm{~mm}$ of diameter, which has a refractive index lower than the core material. The fiber also has a polyethylene coating, which results in a fiber total diameter of $2.2 \mathrm{~mm}$.

The reference works that report the temperature influence on the sensor response employ temperature variation of about $50^{\circ} \mathrm{C}$ for the curvature analysis Bilro et al. (2011), Peters (2011). Since the POF power variation on the temperature range tested is not too large, temperatures within the comfort zone range for wearable applications (Moreno et al., 2008) will not lead to high errors on the sensor response. The comfort zone for wearable applications, as defined in (Moreno et al., 2008), is within the interval between $25^{\circ} \mathrm{C}$ and $45^{\circ} \mathrm{C}$, which is the range applied on the temperature tests. A sample with the sensitive zone parameters aforementioned is positioned inside a heater without curvatures on the sensor fiber. To eliminate movement of the POF sensor during the heating process, the fiber is attached on a plane surface. The heating temperature is controlled and monitored with a calibrated thermocouple temperature sensor positioned close to the POF curvature sensor. A fiber with the same properties is positioned outside the heater to provide the reference signal.

For the humidity variation tests, the same sample employed on the temperature tests is positioned inside a transparent box. This box is sealed and the output of an air humidifier is inserted in the box. A reference fiber, with the same characteristics, is positioned outside the box. The humidity inside the box is measured through the DHT-11 (D-Robotics, United Kingdom) humidity and 
temperature sensor. The test comprises of increase the humidity inside the box and monitoring if it will result on deviations of the POF sensor response. The response is compared with the one of the fiber outside the box to isolate the effects of light source power deviations. The range of this test is $48 \%$ to $94 \%$ due to environmental and operational restrictions, respectively. Since wearable devices such as wearable sensors and exoskeletons can lead to variations of the environmental conditions in the interface between the device and skin (Moreno et al., 2008), the entire test range is of interest for the proposed application.

To obtain a condition of light source power deviation, controlled axial displacements are made between the light coupler and the laser with the POF sensor on a static position. The variation of the sensor response is acquired by the photodiode. The axial displacements are controlled by a micrometer attached on a mechanism with translation stage controlled by the micrometer position as presented in Figure 1.

Figure 2 shows the block diagram of the experiments for the assessment of the influence of light source power and environmental variations on POF power variation. The reference fiber is not under significant environmental (humidity and temperature) variations, whereas the sensor fiber is under temperature, humidity variations. Furthermore, the axial displacement is provoked on the sensor fiber to create an attenuation that emulates the condition of light source power deviation. Each of the environmental tests have a 1-hour duration in to have system stabilization on a time range that are within wearable applications.

\section{Compensation technique analysis}

The light coupler applied on the compensation technique employs the macrobend coupling principle between two fibers. Similar principle is applied on some POF sensor applications (Zhang et al., 2015). It comprises of a twisted-pair of POF with fixed length. The pair of POFs applied has the same dimensions and properties of the sensor fiber, which are PMMA fiber core with diameter of $0.98 \mathrm{~mm}$. The cladding region is made of a fluorinated polymer. Furthermore, the fiber has a step index profile with refractive index of 1.49.

The operation principle of the light coupler employed on the compensation technique is shown in Figure 3. The fibers are twisted and the core of the fibers are very close to each other. The fiber in which the light enters is referred from now on as the active fiber, whereas the fiber in which the light is coupled is the passive fiber. The laser source provides the light for the active fiber, which suffers the macrobending radiation losses due to its low curvature radius. This also results in the polarization of the evanescent wave and the proximity between the

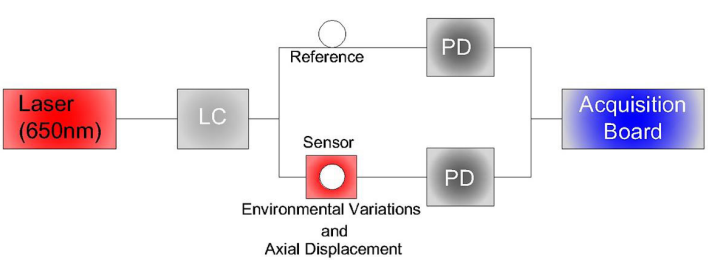

Figure 2. Block diagram for the tests with environmental and light source power variations. PD refers to the photodiode, whereas LC is the light coupler.

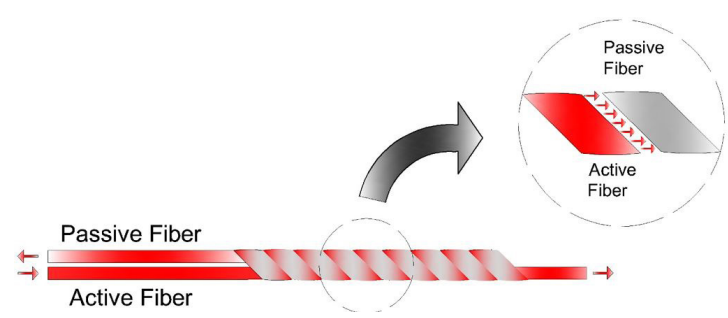

Figure 3. Principle of operation of the compensation technique. The light enters on the active fiber and is coupled with the passive fiber on the twisted region.

cores of the active and passive fibers enable the light energy transference from the active to the passive fiber.

Some commercial light couplers employ the same operation principle. Nevertheless, it has a strict control of the coupling length and optical power source wavelength due to the necessity of obtain an exact split ratio. The coupling region of the light coupler employed has a length of $75 \mathrm{~mm}$, whereas the wavelength employed is $650 \mathrm{~nm}$.

Considering the Port $\mathrm{A}$ as the input port and ports $\mathrm{B}$ and $\mathrm{C}$ as the output ports. Where port $\mathrm{B}$ is the sensor output and Port $\mathrm{C}$ is the reference output, the compensation technique proposed is obtained by a simple normalization of Port B with respect to Port C, as presented in Equation 1. Where $\left(P / P_{0}\right)_{B}$ is the normalized power of Port $\mathrm{B},\left(P / P_{0}\right)_{C}$ is the normalized POF power of Port $\mathrm{C}$ and $\left(P / P_{0}\right)_{\text {comp }}$ is the compensated response. Therefore, the POF power measured in Port $B$ refers to the power attenuation due to angle variation and external conditions e.g. temperature, humidity and light source power variations. Port C, on the other hand, is not submitted to curvatures, which means that its power variation is only due to the variation of external conditions. For this reason, the subtraction between the normalized POF powers measured in Port $\mathrm{B}$ and $\mathrm{C}$ results in a compensated response, where the power variation is due to the angle without the effects of temperature, humidity and light source deviations.

$\left(\frac{P}{P_{0}}\right)_{\text {comp }}=\left(\frac{P}{P_{0}}\right)_{B}-\left(\frac{P}{P_{0}}\right)_{C}$

Characterization of the light coupler employed on the compensation technique is performed by measuring the optical power of the light source and measuring the 
power of each of the ports of the light coupler presented in Figure 3. The power measurements are made with the photodiodes with the transimpedance amplifier presented in Figure 1 without any curvature and axial displacement on the fiber. To validate the compensation technique, different axial displacements are applied on the light coupler through the experimental setup presented in Figure 1. The normalization between the light coupler ports is made and the sensor signal variation is acquired and analyzed to evaluate the technique feasibility. After that, flexion/extension cycles are made to evaluate the POF curvature sensor performance with the proposed compensation technique for light source power deviations.

\section{Results}

\section{Temperature}

Figure 4 presents the results of the temperature test. The sensor response is shown with respect to its power attenuation. The variations of the reference fiber response may be due to the temperature variation outside the heater. However, the variation of the reference fiber response is lower than 0.002 and is related to the decrease of less than $2^{\circ} \mathrm{C}$ of the ambient temperature during the 1 -hour test of temperature variation inside the heater.

\section{Humidity}

On the humidity tests performed, the output signal does not vary significantly with the increase of the humidity. To verify the test repeatability, three tests were made and the same behavior was observed. The fiber responses inside and outside the box are presented in Figure 5.

\section{Light source power deviation}

To simulate different light power source deviations and its influence on the sensor response, the experiment setup presented in Figure 1 is applied without submit the sensor to curvatures, since the goal of this test is to evaluate the influence of the light source deviation on the sensor response.

Figure 6 presents the sensor response for axial displacements of $2 \mathrm{~mm}, 4 \mathrm{~mm}, 6 \mathrm{~mm}$ and $10 \mathrm{~mm}$. To obtain a better visualization of the response, it is shown the time of the experiment and the POF sensor signal attenuation at each displacement tested. The errors of the sensor at each displacement are also presented

\section{Compensation technique}

A simple procedure is applied to characterize the light coupler ports. The laser optical power is measured with the photodiode. Thereafter, the photodiode is positioned on the output ports (Port B and Port C). The results of the coupling effect between the fibers and ports positions are presented in Figure 7a.

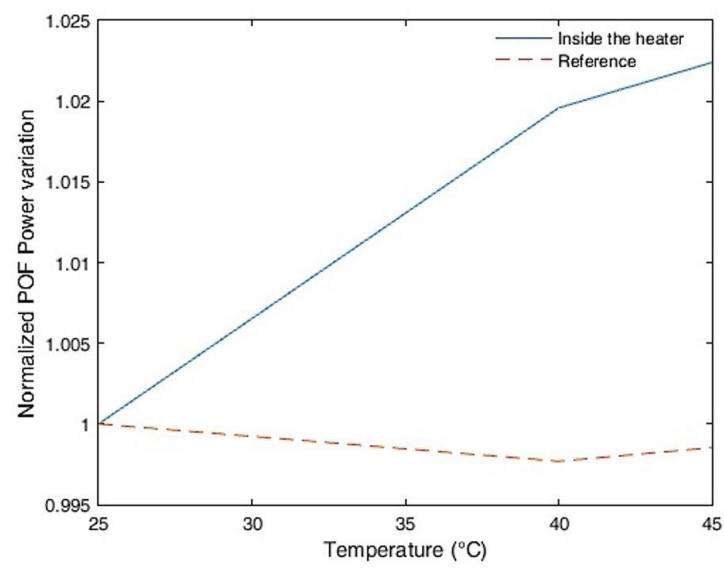

Figure 4. Normalized POF response as a function of temperature variation. Blue curve represents the POF inside the heater, whereas the red curve is the sensor outside the heater (reference).

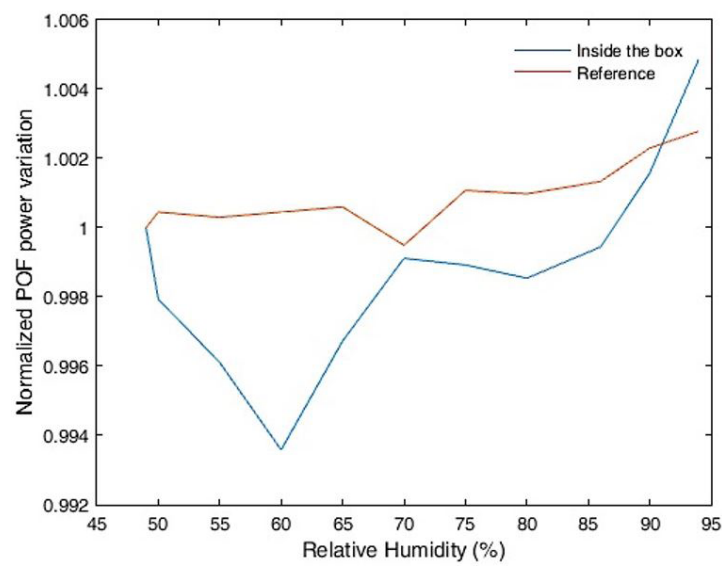

Figure 5. Normalized POF response as a function of humidity variation. Blue curve represents the POF inside the box, whereas the red curve is the sensor outside the box (reference).

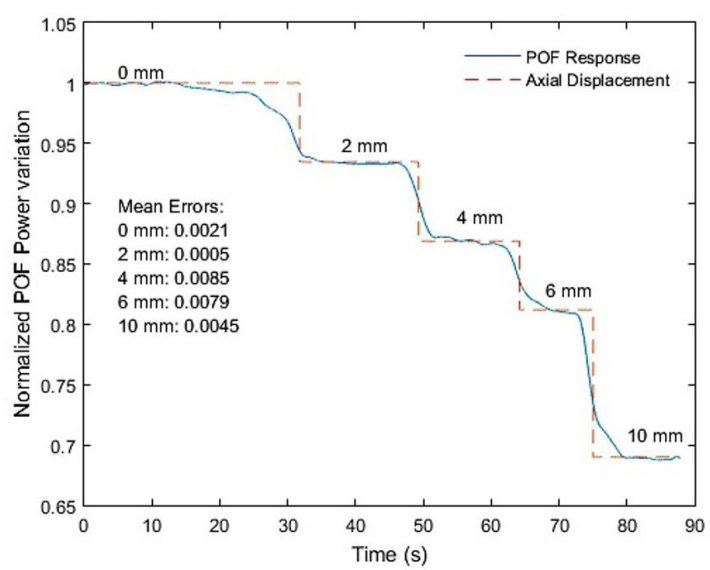

Figure 6. Normalized POF response as a function of light source power variation. The variation of the light source is obtained by applying different axial displacements between the laser and the fiber. 


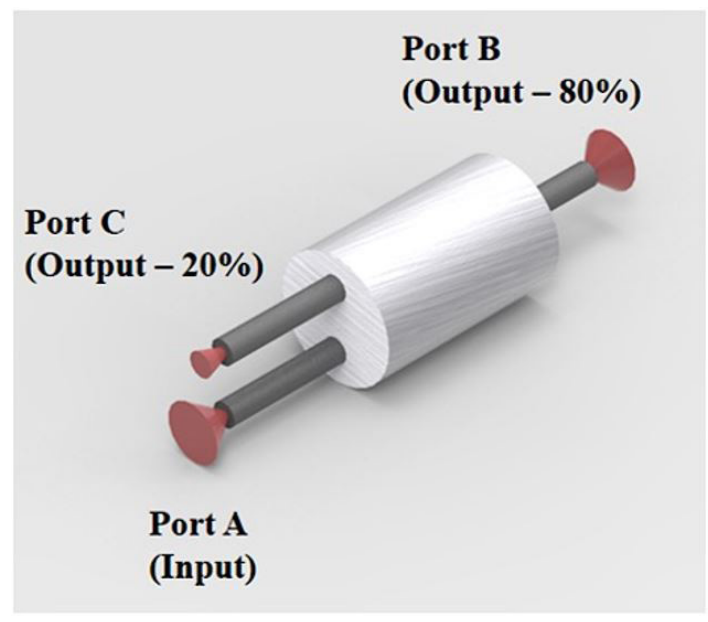

(a)

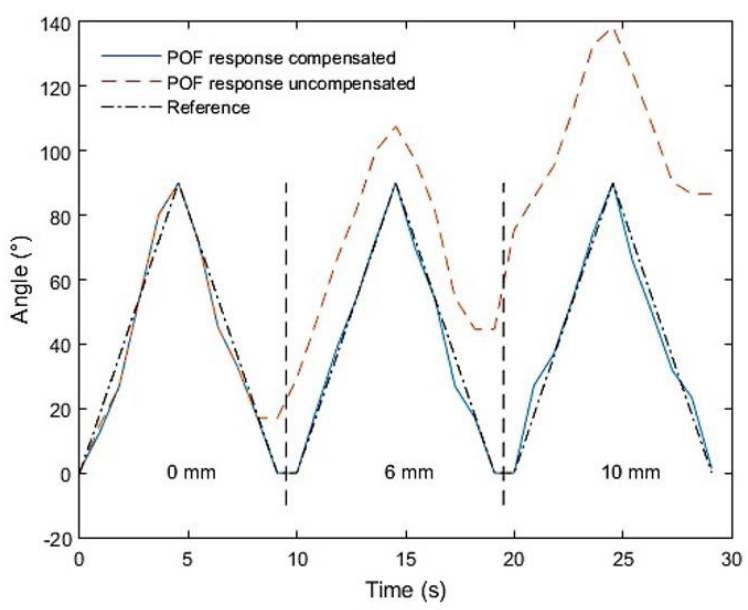

(b)

Figure 7. (a) Light coupler characterization. The light enters on the input port of the active fiber and $20 \%$ of the light power is coupled to the passive fiber (Port C). The remaining light ( $80 \%$ ) exits on the other end of the active fiber. (b) POF response with the light source power variation with and without the compensation technique on flexion/extension cycles.

After the light coupler characterization, the compensation technique is validated on flexion and extension cycles with the sensor. To prove the feasibility of the sensor and the compensation technique, 3 different axial displacements are applied to create a power variation. Figure $7 \mathrm{~b}$ shows the POF sensor response for dynamic tests with constant angular velocity on the test prototype presented in Figure 1 with and without the application of the compensation technique proposed.

Additional experiments were performed in conditions closer to the real application of the curvature sensor on wearable devices for knee angle assessment and are presented in Figure 8. The conditions for the last set of experiments are flexion/extension cycles without the positioner for axial displacements presented in Figure 1. Furthermore, the movements are made on a single plane, which is similar to the knee movement that occurs mainly on the sagittal plane (Kirtley, 2006).

\section{Discussion}

For the temperature tests (see Figure 4), the range defined for wearable devices application is between $25^{\circ} \mathrm{C}$ and $45^{\circ} \mathrm{C}$, which is within the interval of thermal comfort presented in (Moreno et al., 2008). Since the sensitivity of the sensor with respect to the angle is higher than $0.05 \mathrm{deg}^{-1}$, which is the one obtained for the same sensor in Leal-Junior et al. (2018), the variation obtained on the range of interest represent an equivalent angular error lower than $0.5^{\circ}$. Therefore, for the range of interest, the temperature effect on the POF curvature sensor response does not lead to significant errors on the sensor angle measurement.

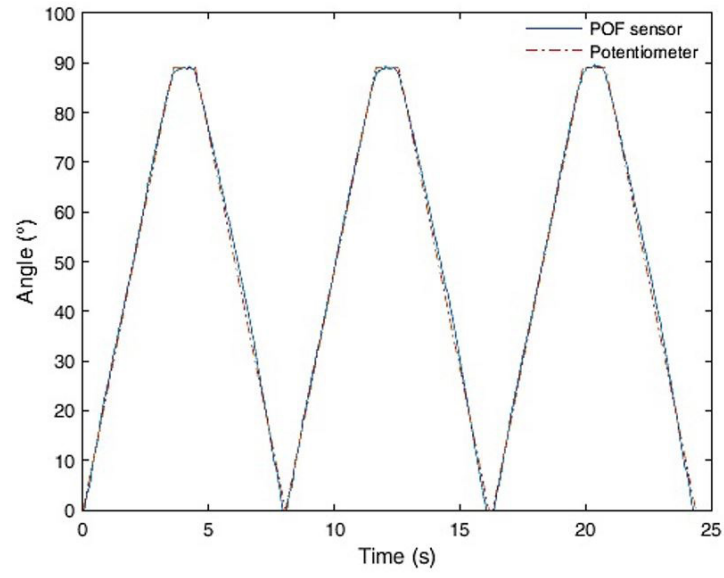

Figure 8. POF sensor response with the compensation technique and potentiometer response to dynamic bending tests.

The POF response with respect to the relative humidity variation is also shown with respect to the fiber attenuation. Since the two fibers present approximately the same length and sensitive zone parameters, both signals present similar amplitudes. The (normalized) difference between the maximum and minimum response of the sensor inside the box is about 0.011 , which would lead to an angular error of about $0.2^{\circ}$. Therefore, the humidity variation also not lead to significant errors on the POF based curvature sensor.

Experimental results demonstrated that, for this specific POF sensor, the variation of temperature and humidity within the ranges of wearable devices applications do not lead to significant sensor response deviations. Therefore, 
in this case, a compensation technique or setup for temperature and humidity variations is not necessary.

However, results show that a light source power variation leads to higher errors on the POF sensor response and a compensation of this effect is required. A common compensation technique is based on a reference fiber that remains on a static position during the measurement. For this purpose, Bilro et al. (2011) and Moraleda et al. (2013) proposed the use of a reference fiber in the same direction of the POF sensor. Such solution may not be suitable for wearable applications, since this reference fiber may move or bend with the POF sensor, presenting signal attenuations that could result in measurement errors. Alternatively, mechanical isolation on the reference fiber could be provided by additional mechanisms, which harms the device compactness and desirable simplicity. To overcome this limitation, a different light coupler was proposed in this work, which presents a configuration that the reference signal is not on the curvature region of the POF sensor.

After the light coupler characterization, flexion and extension cycles were made on the sensor with the test prototype presented in Figure 1. The flexion and extension cycles are made under the condition of induced light power variation by increasing the axial displacement between the light source and the fiber coupler. To illustrate the effects of the proposed compensation technique, results are shown with (solid line in Figure $7 \mathrm{~b}$ ) and without (dashed line in Figure 7b) the application of the compensation technique. It is possible to observe that the presented setup is suitable even for power variations such as the one presented in Figures 6 and $7 b$, which are much higher than the fluctuations found on conventional optical sources. Therefore, the proposed technique is suitable for compensating not only minor variations due to light source deviations, but also errors that may arise from major misalignments between the light source and the POF sensor, since Figure 7(b) shows that the proposed technique is able to compensate misalignments as high as $10 \mathrm{~mm}$. This is a compensated range higher than the one presented in (Zawawi et al., 2013) and equal to the one presented in (Vallan et al., 2012) with the additional advantage of employing only one light source that results in a simpler design of the sensor and lower cost. This is especially desirable in wearable applications, where such misalignments may occur due to the dynamic nature of the human motion. In this manner, there are also gains regarding the robustness of the sensor to external mechanical perturbations and the simplicity of implementation, avoiding unnecessary recalibrations.

Experiments, presented in Figure 8, revealed a root mean squared error (RMSE) of approximately $1^{\circ}$ when comparing the developed POF sensor with the reference (precision) potentiometer. This value is lower than the errors obtained with IMUs (Vargas-Valencia et al., 2016) with the additional advantages of low cost, easiness of implementation and greater simplicity on signal processing. Comparing with other POF-based curvature sensors based on the same operation principle, the sensor with the developed compensation technique present smaller errors than the ones found in Bilro et al. (2011), Donno et al. (2008) and Stupar et al. (2012), which are $2^{\circ}, 1.36^{\circ}$ and $2.5^{\circ}$, respectively. The lower errors of the sensor proposed in this work are due to the compensation technique applied. In addition, the errors are similar to or lower than the ones obtained in Leal-Junior et al. (2018) and Leal-Junior et al. (2017b). Comparing with commercially available devices, the proposed POF curvature sensor presents errors lower than electrogoniometers (Piriyaprasarth and Morris, 2007) and IMUs (Vargas-Valencia et al., 2016). The authors emphasize that such errors can be further reduced with the analysis and compensation of the polymer viscoelastic response, which will be explored on future works by means of an analysis of the POF material properties and characterization of the viscoelastic response that leads to errors on the POF curvature sensor measurements (Leal-Junior et al., 2017a), where such reduction of the errors can provide a more reliable sensor for human movement analysis and wearable robots applications. Nevertheless, the proposed sensor is a low-cost and portable solution with errors close to or lower than the ones of the conventional technologies for wearable applications such as IMUs and electrogoniometers.

\section{Acknowledgements}

This research is supported by CAPES (88887.095626/2015-01), FAPES (72982608), CNPq (304192/2016-3) and Petrobras (23068.013529/2012-56).

\section{References}

Antunes PF, Varum H, Andre PS. Intensity-encoded polymer optical fiber accelerometer. IEEE Sens J. 2013; 13(5):1716-20. http://dx.doi.org/10.1109/JSEN.2013.2242463.

Bilro L, Oliveira JG, Pinto JL, Nogueira RN. A reliable low-cost wireless and wearable gait monitoring system based on a plastic optical fibre sensor. Meas Sci Technol. 2011; 22(4):45801. http://dx.doi.org/10.1088/0957-0233/22/4/045801.

Bilro L, Alberto N, Pinto JL, Nogueira R. Optical sensors based on plastic fibers. Sensors (Basel). 2012; 12(9):12184207. PMid:23112707. http://dx.doi.org/10.3390/s120912184.

Bolink SAAN, Naisas H, Senden R, Essers H, Heyligers IC, Meijer K, Grimm B. Validity of an inertial measurement unit to assess pelvic orientation angles during gait, sit-stand transfers and stepup transfers: comparison with an optoelectronic motion capture system. Med Eng Phys. 2016; 38(3):225-31. PMid:26711470. http://dx.doi.org/10.1016/j.medengphy.2015.11.009. 
Braidot AA, Cifuentes CC, Frizera A No, Frisoli M, Santiago A. ZigBee wearable densor development for upper limb robotics rehabilitation. IEEE Lat Am Trans. 2013; 11(1):408-13. http:// dx.doi.org/10.1109/TLA.2013.6502838.

Coulthard JT, Treen TT, Oates AR, Lanovaz JL. Evaluation of an inertial sensor system for analysis of timed-up-and-go under dual-task demands. Gait Posture. 2015; 41(4):882-7. PMid:25827680. http://dx.doi.org/10.1016/j.gaitpost.2015.03.009.

Dejnabadi H, Jolles BM, Aminian K. A new approach to accurate measurement of uniaxial joint angles based on a combination of accelerometers and gyroscopes. IEEE Trans Biomed Eng. 2005; 52(8):1478-84. PMid:16119244. http:// dx.doi.org/10.1109/TBME.2005.851475.

Donno M, Palange E, Di Nicola F, Bucci G, Ciancetta F. A new flexible optical fiber goniometer for dynamic angular measurements: Application to human joint movement monitoring. Instrumentation. 2008; 57(8):1614-20. http:// dx.doi.org/10.1109/TIM.2008.925336.

Dunne LE, Walsh P, Hermann S, Smyth B, Caulfield B. Wearable monitoring of seated spinal posture. IEEE Trans Biomed Circuits Syst. 2008; 2(2):97-105. PMid:23852756. http://dx.doi.org/10.1109/TBCAS.2008.927246.

El-Gohary M, McNames J. Shoulder and elbow joint angle tracking with inertial sensors. IEEE Trans Biomed Eng. 2012; 59(9):2635-41. PMid:22911538. http://dx.doi.org/10.1109/ TBME.2012.2208750.

Holland D, Abah C, Velasco-Enriquez M, Herman M, Bennett GJ, Vela EA, Walsh CJ. The soft robotics toolkit. IEEE Robot Autom Mag. 2017; 24(1):57-64. http://dx.doi.org/10.1109/ MRA.2016.2639067.

Kirtley K. Clinical gait analysis: theory and practice. Washington: Churchill Livingstone; 2006.

Leal-Junior AG, Frizera-Neto A, Pontes MJ. Analytical model for a polymer optical fiber under dynamic bending. Opt Laser Technol. 2017a; 93:92-8. http://dx.doi.org/10.1016/j. optlastec.2017.02.009.

Leal-Junior AG, Frizera-Neto A, Pontes MJ, Botelho TR. Hysteresis compensation technique applied to polymer optical fiber curvature sensor for lower limb exoskeletons. Meas Sci Technol. 2017b; 28(12):1-9. http://dx.doi.org/10.1088/13616501/aa946f.

Leal-Junior AG, Frizera-Neto A, Pontes MJ. Sensitive zone parameters and curvature radius evaluation for polymer optical fiber curvature sensors. Opt Laser Technol. 2018; 100:272-81. http://dx.doi.org/10.1016/j.optlastec.2017.10.006.

Liu R, Zhuang F, Yanzheng Z, Qixin C, Shuguo W. Operation principle of a bend enhanced curvature optical fiber sensor. IEEE Int Conf Intell Robot Syst. 2006; 1966-71.

Marques CA, Peng GD, Webb DJ. Highly sensitive liquid level monitoring system utilizing polymer fiber Bragg gratings. Opt Express. 2015; 23(5):6058-72. PMid:25836830. http://dx.doi. org/10.1364/OE.23.006058.

Moraleda AT, García CV, Zaballa JZ, Arrue J. A temperature sensor based on a polymer optical fiber macro-bend. Sensors (Basel). 2013; 13(10):13076-89. PMid:24077323. http://dx.doi. org/10.3390/s131013076.
Moreno JC, Bueno L, Pons JL. Wearable robot technologies. In: Pons JL, editor. Wearable robots: biomechatronic exoskeletons. Hoboken: John Wiley \& Sons; 2008. p. 165-99.

Peters K. Polymer optical fiber sensors - a review. Smart Mater Struct. 2011; 20(1):13002. http://dx.doi.org/10.1088/09641726/20/1/013002.

Piriyaprasarth P, Morris ME. Psychometric properties of measurement tools for quantifying knee joint position and movement: A systematic review. Knee. 2007; 14(1):2-8. PMid:17140797. http://dx.doi.org/10.1016/j.knee.2006.10.006.

Rhea MR, Kenn JG, Peterson MD, Massey D, Simão R, Marin PJ, et al. Joint-angle specific strength adaptations influence improvements in power in highly trained athletes. Hum Mov. 2016; 17(1):43-9.

Rodriguez-Cobo L, Lomer M, Cobo A, Lopez-Higuera J. Optical fiber strain sensor with extended dynamic range based on specklegrams. Sens Actuators A Phys. 2013; 203:341-5. http://dx.doi.org/10.1016/j.sna.2013.09.006.

Stupar DZ, Bajic JS, Manojlovic LM, Slankamenac MP, Joza $\mathrm{V}$, Zivanov MB. Wearable low-cost system for human joint movements monitoring based on fiber-optic curvature sensor. IEEE Sens J. 2012; 12(12):3424-31. http://dx.doi.org/10.1109/ JSEN.2012.2212883.

Tong K, Granat MH. A practical gait analysis system using gyroscopes. Med Eng Phys. 1999; 21(2):87-94. PMid:10426508. http://dx.doi.org/10.1016/S1350-4533(99)00030-2.

Vallan A, Casalicchio ML, Olivero M, Perrone G. Assessment of a dual-wavelength compensation technique for displacement sensors using plastic optical fibers. IEEE Trans Instrum Meas. 2012; 61(5):1377-83. http://dx.doi.org/10.1109/TIM.2011.2180975.

Vargas-Valencia LS, Elias A, Rocon E, Bastos-Filho T, Frizera A. An IMU-to-body alignment method applied to human gait analysis. Sensors (Basel). 2016; 16(12):2090. PMid:27973406. http://dx.doi.org/10.3390/s16122090.

Villa-Parra AC, Broche L, Delisle-Rodriguez D, Sagaró R, Bastos T, Frizera-Neto A. Design of active orthosis for a robotic gait rehabilitation system. Front Mech Eng. 2015; 10(3):242-54. http://dx.doi.org/10.1007/s11465-015-0350-1.

Wang PT, King CE, Do AH, Nenadic Z. A durable, low-cost electrogoniometer for dynamic measurement of joint trajectories. Med Eng Phys. 2011; 33(5):546-52. PMid:21247789. http:// dx.doi.org/10.1016/j.medengphy.2010.12.008.

Williams JM, Haq I, Lee RY. Dynamic measurement of lumbar curvature using fibre-optic sensors. Med Eng Phys. 2010; 32(9):1043-9. PMid:20678954. http://dx.doi.org/10.1016/j. medengphy.2010.07.005.

Yuan L. Automatic-compensated two-dimensional fiber-optic sensor. Opt Fiber Technol. 1998; 4(4):490-8. http://dx.doi. org/10.1006/ofte.1998.0266.

Zawawi MA, O'Keeffe S, Lewis E. Plastic optical fibre sensor for spine bending monitoring with power fluctuation compensation. Sensors (Basel). 2013; 13(11):14466-83. PMid:24233073. http://dx.doi.org/10.3390/s131114466.

Zhang H, Feng L, Hou Y, Su S, Liu W, Liu J, Xiong J. Optical fiber liquid level sensor based on macro-bending coupling. Opt Fiber Technol. 2015; 24:135-9. http://dx.doi.org/10.1016/j. yofte.2015.05.012. 